\title{
The clinical effects of a new management mode for hypertensive patients: a randomized controlled trial
}

\author{
Yan-Qin Sun, Yong-Ping Jia, Ji-Yuan Lv, Gui-Jin Ma \\ Department of Cardiovascular Medicine, The First Hospital of Shanxi Medical University, Taiyuan, China \\ Contributions: (I) Conception and design: YQ Sun; (II) Administrative support: YP Jia; (III) Provision of study materials or patients: JY Lv, GJ Ma; \\ (IV) Collection and assembly of data: YQ Sun, JY Lv, GJ Ma; (V) Data analysis and interpretation: YP Jia; (VI) Manuscript writing: All authors; (VII) \\ Final approval of manuscript: All authors. \\ Correspondence to: Yong-Ping Jia, PhD. Department of Cardiovascular Medicine, The First Hospital of Shanxi Medical University, No. 85 of Jiefang \\ South Road, Yingze District, Taiyuan 030001, China. Email: drjiajyp1428@163.com.
}

Background: The Internet, smartphones, and the application of health technology have great potential for hypertension management. We aim to evaluate a new mode of mobile health management with a social network application to guide blood pressure management in patients with hypertension.

Methods: Using a randomized controlled trial design, 120 hypertensive patients in the First Hospital of Shanxi Medical University who volunteered to participate in the study were randomly divided into an experimental group and a control group. The experimental group was divided into low, middle, or highrisk groups according to the cardiovascular risk stratification. The blood pressures of both the experimental group (the WeChat-guided new mode group) and the control group (the conventional mode group) were administered for three months.

Results: With intervention, both systolic blood pressure (SBP) and diastolic blood pressure (DBP) in the experimental group were significantly lower than those in the control group $(\mathrm{P}=0.016)$. The SBP and DBP of the experimental group after intervention were lower than those before intervention $(\mathrm{P}<0.001)$, which was not observed in the control group $(\mathrm{P}=0.056)$. There was no difference in the SBP drops in the low-risk, middle-risk, and high-risk groups $(\mathrm{P}=0.402)$. Similarly, no difference in DBP drop was observed $(\mathrm{P}=0.628)$. There were no differences in Colorado Pretrial Assessment Tool (CPAT) scores between the experimental group and the control group before intervention $(\mathrm{P}=0.509)$. After intervention, CPAT scores in the experimental group were higher than those in the control group $(\mathrm{P}<0.001)$. Before intervention, there was no significant difference in the Hypertension Patients Self-Management Behavior Rating Scale (HPSMBRS) scores, blood lipid, body mass index (BMI), and urinary microalbumin between the experimental group and the control group $(\mathrm{P}>0.05)$. After intervention, the HPSMBRS score in the experimental group was significantly higher than that in the control group $(\mathrm{P}<0.05)$. The HPSMBRS score of the experimental group after intervention was higher than before intervention, and BMI, urinary microalbumin, TC, LDL-C were lower than before intervention $(\mathrm{P}<0.05)$.

Conclusions: This new mode of mobile health management has a good effect on blood pressure control in patients with hypertension. It provides evidence for the application of mobile information technology for hypertension patients in clinical practice.

Keywords: Mobile health; management mode; hypertension; WeChat; China

Submitted Jun 28, 2020. Accepted for publication Nov 01, 2020.

doi: $10.21037 / \mathrm{cdt}-20-589$

View this article at: http://dx.doi.org/10.21037/cdt-20-589 


\section{Introduction}

Hypertension is a global public health problem and a major risk factor for cardiovascular and cerebrovascular diseases. It has become a major factor in the global burden of disease (1). Currently, about one billion people worldwide suffer from high blood pressure (2-4). In recent years, the prevalence of hypertension has increased rapidly in China, reaching $25.2 \%$ among adults (5). The awareness rate of hypertension was $51.5 \%$, the treatment rate was $46.1 \%$, and the control rate was $16.9 \%$ (6). The studies demonstrate that daily lifestyle is one of the main factors affecting the blood pressure control levels of patients with hypertension, and the combination of drugs and lifestyle intervention is conducive to the prevention and treatment of hypertension (7-10). The conventional face-to-face management mode of chronic diseases has high human and material costs and is difficult to maintain and cover (11).

With the rapid development of networking and mobile Internet use, mobile information technology provides opportunities for online medical services (12). WeChat (Wei Xin), a form of mobile information technology, was released in 2011 and is a communication platform developed by the Tencent group. We can use it to send voice and text messages, make free phone calls, and subscribe to public accounts to get a wide variety of up-to-date information (13). In 2017, WeChat accounted for $86.07 \%$ of social software used by the elderly in China $(14,15)$. All kinds of information, including health knowledge that is of great concern to middle-aged and older people, spread rapidly among a large number of users through WeChat. Zhang et al. (16) concluded from their survey that the public has a high level of access to health information through WeChat. WeChat is a new clinical teaching tool based on problem-learning, proposed by Zeng et al. (17).

We used the WeChat platform as a mobile health intervention measure to study the effectiveness and feasibility of this new mode of mobile platform management in patients with hypertension, aiming at improving the blood pressure and self-management behavior of patients with hypertension. We present the following article in accordance with the CONSORT reporting checklist (available at http://dx.doi.org/10.21037/cdt-20-589).

\section{Methods}

\section{Objects}

Hypertensive patients discharged from the First Hospital of
Shanxi Medical University or reexamined in the outpatient department were selected. The study was conducted in accordance with the Declaration of Helsinki (as revised in 2013). The study was approved by ethics board of The First Hospital of Shanxi Medical University (No. sxykdxdyyyll201964) and informed consent was taken from all the patients. The privacy of the patients is protected by law and the personal information of the patients will not be disclosed. They were randomly divided into an experimental group (mean age $52.35 \pm 9.46$ years) with 60 cases and a control group with 60 cases (mean age $53.42 \pm 8.78$ years) by a random number table. The experimental group was divided into a low-risk group (13 cases), an intermediaterisk group (25 cases), and a high-risk group (22 cases), according to the cardiovascular risk stratification (Table 1). There was no significant difference in gender and age between the experimental group and the control group.

\section{Diagnostic criteria and inclusion and exclusion criteria for bypertension}

The diagnosis of hypertension is based on the diagnostic and classification criteria of the latest guidelines for hypertension in China (18). Inclusion criteria: adult hypertensive patients with the ability to use WeChat and with reading and writing skills. Exclusion criteria: patients who had (I) acute cardiovascular and cerebrovascular disease; (II) a history of secondary hypertension, hypertensive crisis or malignant tumor; (III) severe cognitive impairment; (IV) nephropathy or diabetic foot issues, or serious clinical complications requiring hospitalization; (V) arrhythmia; (VI) malignant tumors; (VII) participated in any other studies currently or within a year; or (VIII) poor patient compliance [Colorado Pretrial Assessment Tool (CPAT) score below 2].

\section{Control group management methods}

Patients continued to receive conventional hypertension management. Conventional management models included appropriate antihypertensive drugs according to the latest guidelines for hypertension in China (18), face-to-face education on hypertension, reasonable lifestyle advice, distribution of hypertension health manuals, and selfmonitoring and recording of blood pressure at home. It was recommended that patients record their blood pressure in the morning and before bed two to three times a week. If the blood pressure was well-controlled, the frequency could be reduced to once a week. If the blood pressure was not 
Table 1 Content of mobile health intervention based on WeChat

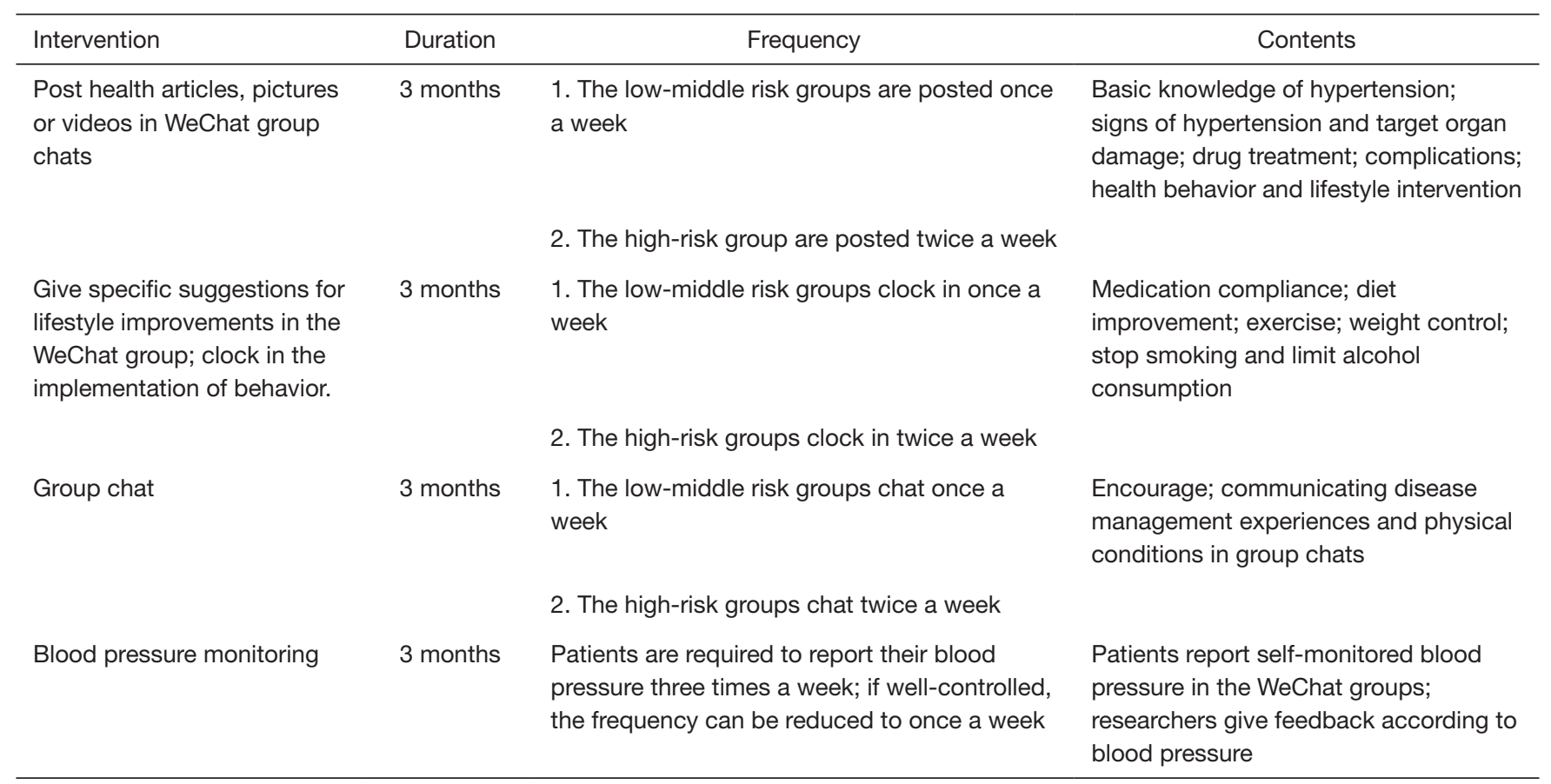

SBP, systolic blood pressure; DBP, diastolic blood pressure.

well-controlled for 2 weeks, patients were advised to see a doctor in the outpatient department. The patients in the low-risk group were followed up every three months, those in the intermediate-risk group were followed up every two months, and those in the high-risk group were followed up every month. Patients were recommended to go to the hospital at any time if there is any discomfort. No additional follow-up intervention was given.

\section{Experimental group management methods}

The experimental group was divided into a low-risk group, a middle-risk group, and a high-risk group according to the cardiovascular risk stratification (Table 1). Three WeChat groups were set up and named "new horizon group of blood pressure lowering", "action of blood pressure lowering", and "small expert of blood pressure lowering", corresponding to the low-risk group, middle-risk group, and high-risk group, respectively.

The intervention lasted for three months, including health education, health behavior promotion, group chats, and BP monitoring. The contents of health education and behavior promotion are mainly based on Chinese Guidelines for the Management of Hypertension, revised for 2018 (the Guidelines) (19). Specific interventions were identified based on the subjects' cardiovascular risk stratification (Table 2). According to the classification of cardiovascular risk, health knowledge articles, questionnaires, pictures, or videos were published in WeChat group chats at a certain frequency. These included basic knowledge of hypertension, signs of hypertension, target organ damage, complications, drug treatment, health behavior, and lifestyle intervention. Participants were asked to perform specific behaviors at a certain frequency and to clock in the WeChat group. Those who actively completed these tasks received verbal encouragement. Those who failed to meet their goals twice in a row were contacted privately to determine the cause. At each intervention, if $60 \%$ of the participants had read a health education article or completed the task, the participation was deemed as have passed. If the percentage reached more than $80 \%$, participation was great. For each subject, if the test score reached 60 , we considered it as an effective intervention; if the score was over 80 , the intervention was considered great. Patients were encouraged to share their experiences in group chats. The group chat was initiated by the researcher. Patients were instructed to take their medication regularly, control their weight, reduce their sodium intake to no more than 6 grams per 
Table 2 Comparison of blood pressure before and after intervention in the two groups

\begin{tabular}{|c|c|c|c|c|c|c|c|c|}
\hline Group & \multicolumn{4}{|c|}{ SBP $(\mathrm{mmHg})$} & \multicolumn{4}{|c|}{$\mathrm{DBP}(\mathrm{mmHg})$} \\
\hline Experimental group & $143.15 \pm 10.13$ & $132.23 \pm 8.86$ & 6.281 & $<0.001$ & $88.30 \pm 8.16$ & $82.62 \pm 6.98$ & 4.100 & $<0.001$ \\
\hline Control group & $142.73 \pm 10.69$ & $139.30 \pm 8.66$ & 1.933 & 0.056 & $88.00 \pm 8.65$ & $85.77 \pm 7.07$ & 1.548 & 0.124 \\
\hline $\mathrm{t}$ & 0.219 & -4.419 & & & 0.195 & -2.455 & & \\
\hline
\end{tabular}

SBP, systolic blood pressure; DBP, diastolic blood pressure.

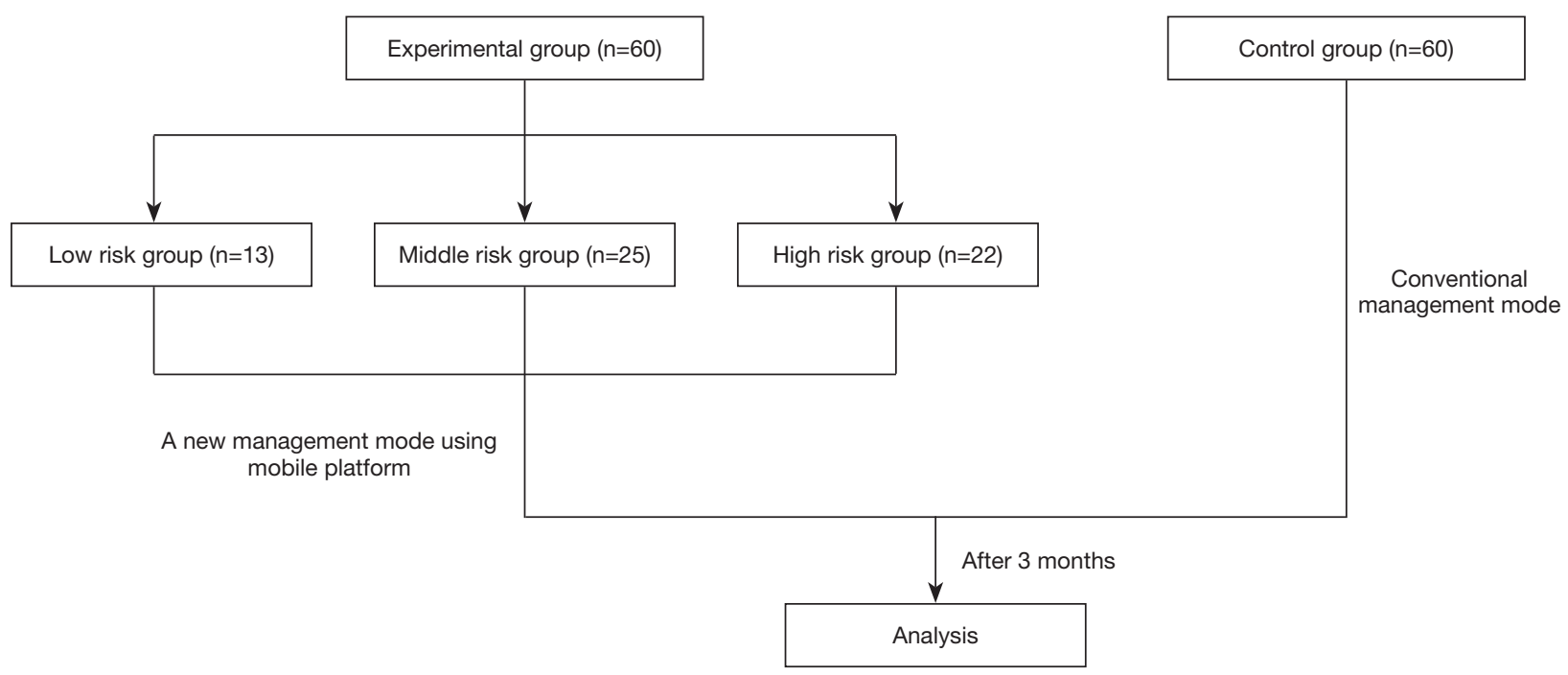

Figure 1 Flow chart of experimental method.

day, reduce their fat intake, eat more fresh vegetables and fruits, quit smoking, take appropriate exercise, and adjust their lifestyle. According to the fluctuation of the patient's blood pressure, patients were advised to adjust the type and dose of antihypertensive drugs and master the appropriate time to take medicine. People could freely share their disease management experiences and physical conditions in group chats. The frequency of group chat varied according to the stratification of cardiovascular risk. Each chat lasted nearly an hour. Participants could also consult researchers privately through WeChat. All subjects in the experimental group were asked to clock in their blood pressure during a group chat. Each person reported their blood pressure three times a week. For patients with well-controlled blood pressure, the reporting frequency was reduced to once a week. If a patient's blood pressure wasn't well-controlled for 2 weeks, the researchers would warn them to seek medical attention. The content of mobile health intervention based on WeChat is shown in Table 1.

A flow chart of the experimental method is shown in Figure 1. The main indexes were systolic blood pressure (SBP), diastolic blood pressure (DBP), blood pressure fluctuation value, lipid profile, body weight, and urinary microalbumin.

\section{Medication compliance}

Patient compliance with treatment for hypertension (CPAT) refers to the extent to which patients with hypertension follow their doctor's recommendations (20). Morisky et al. (21) reported four questions that could be used to determine the subjects' CPAT: (I) Have you ever forgotten to take your medication? (II) Do you not take medicine seriously sometimes? (III) Have you ever stopped taking medication when your symptoms improved? (IV) Have you ever stopped taking medication when you became more 
Table 3 Hypertension cardiovascular risk stratification

\begin{tabular}{lccc}
\hline Risk factors and medical history & $\begin{array}{c}\text { Hypertension grade 1, } \\
\text { SBP 140-159/DBP 90-99 }\end{array}$ & $\begin{array}{c}\text { Hypertension grade 2, } \\
\text { SBP160-179/DBP 100-109 }\end{array}$ & $\begin{array}{c}\text { Hypertension grade 3, } \\
\text { SBP } \geq 180 / D B P \geq 110\end{array}$ \\
\hline None & Low risk & Middle risk & High risk \\
$1-2$ risk factors & Middle risk & Middle risk & High risk \\
$\geq 3$ risk factors or complications & High risk & High risk & High risk \\
\hline
\end{tabular}

SBP, systolic blood pressure; DBP, diastolic blood pressure. Risk factors: male $>55$ years old, female >65 years old; dyslipidemia; smoking; family history of early onset of cardiovascular disease; obesity (BMI $\geq 28 \mathrm{~kg} / \mathrm{m}^{2}$ ). Complications: cerebrovascular disease, heart disease, kidney disease, peripheral vascular disease, retinopathy, diabetes.

ill? The answers to these four questions are called CPAT scores. The score for "yes" is 0 , and the score for "no" is 1 . The cumulative scores were defined as follows: high CPAT was 4 , medium CPAT was $2-3$, and low CPAT was $0-1$.

\section{Hypertension Patients Self-Management Bebavior Rating Scale (HPSMBRS)}

Hypertension self-management behaviors were measured by HPSMBRS for hypertension patients designed by Zhao et al. (22) in 2012. It consists of six management dimensions (medication, disease monitoring, diet, exercise, work and rest, and emotion), with a total of 33 items. Each item is rated as "always, often, sometimes, rarely, never" using a Likert 5 scale, with a score of 5 to 1 . The higher the score is, the higher the level of self-management is. In order to make the dimensions comparable, the scores of each dimension are converted into standard scores. Standard score $=($ actual scores of each factor/total scores of each factor) $\times 100$. Cronbach's $\alpha$ coefficients for the subscale and the total scale were $0.757-0.911$ and 0.914 , respectively. According to the standardized grading standard of selfmanagement level, the self-management level is divided into three grades: low ( $<60$ points), medium ( $60-80$ points) and high ( $>80$ points). This scale has good reliability, validity and operability, and has been widely used to evaluate the self-management of hypertension patients (22).

\section{Statistical analysis}

SPSS20.0 statistical software (IBM, USA) was used for analysis. First, the Kolmogorov-Smirnov test was performed on variables for normality. For data with normal distribution, the mean standard deviation was adopted for continuous parameter variables. A $t$-test was used in the two groups. Analysis of variance was used to compare the blood pressure drop values of the three subgroups in the experimental group, and an LSD $t$-test was used for further pairwise comparison. For data with non-normal distribution, the median was used. A rank-sum test was used in the two groups. A Kruskal-Wallis test was used to compare the blood pressure drop values of the three subgroups in the experimental group, and a Bonferroni test was used for further pairwise comparison. Chi-square tests were used to compare qualitative data among the groups. Two-tailed $\mathrm{P}$ value $<0.05$ was considered statistically significant.

\section{Results}

Baseline data analysis: the total number of samples in the experimental group was 60 , including 32 males and 28 females. One of the male patients asked to withdraw from the experiment after two months, so the number of valid samples in the experimental group was 59 . The total number of samples in the control group was 60, including 29 males and 31 females. Among them, one male patient and one female patient lost contact, so the effective sample size of the control group was 58 . There were no statistically significant differences in age, gender, diabetes history, smoking history, blood pressure classification, or types of antihypertensive drugs between the experimental group and the control group, and the experimental group and the control group were comparable (Table 3).

Comparative analysis before and after intervention: The experimental group adopted the new management mode of mobile platform management (WeChat) for three months, while the control group adopted the conventional management mode. Blood pressure values before and after intervention in the two groups were shown in Table 4. SBP and DBP before intervention were compared between the experimental group and the control group, and the 
Table 4 Comparison of general clinical data between the two groups

\begin{tabular}{|c|c|c|c|}
\hline Clinical indicators & Experimental group (59 cases) & Control group (58 cases) & $\mathrm{P}$ \\
\hline Age, years $(\bar{x} \pm s)$ & $52.35 \pm 9.46$ & $53.42 \pm 8.78$ & 0.523 \\
\hline Smoke (yes/no) & $32 / 27$ & $28 / 30$ & 0.465 \\
\hline Diabetes (yes/no) & $12 / 47$ & $9 / 49$ & 0.487 \\
\hline CKD (yes/no) & $2 / 57$ & $1 / 57$ & 0.619 \\
\hline Heat failure (yes/no) & $3 / 56$ & $1 / 57$ & 0.679 \\
\hline Blood pressure levels ( 1 and 2 level/3 level) & $44 / 15$ & $46 / 12$ & 0.666 \\
\hline Anti-hypertension drugs (0/1-2/>2 kinds) & $4 / 35 / 20$ & $2 / 44 / 12$ & 0.208 \\
\hline
\end{tabular}

$\mathrm{CHD}$, coronary heart disease; CKD, chronic kidney disease.

Table 5 Comparison of subgroup blood pressure drop value before and after intervention in the experimental group

\begin{tabular}{lcc}
\hline Group & Decreased value SBP $(\mathrm{mmHg})$ & Decreased value DBP $(\mathrm{mmHg})$ \\
\hline Low risk group & $8.77 \pm 5.57$ & $4.69 \pm 5.22$ \\
Middle risk group & $10.80 \pm 8.16$ & $6.40 \pm 5.26$ \\
High risk group & $12.32 \pm 7.59$ & $5.45 \pm 5.49$ \\
F & 0.927 & 0.470 \\
P & 0.402 & 0.628 \\
\hline
\end{tabular}

SBP, systolic blood pressure; DBP, diastolic blood pressure.

difference was not statistically significant $(\mathrm{P}>0.05)$. After intervention, SBP and DBP in the experimental group were lower than those in the control group, with statistically significant differences $(\mathrm{P}<0.001)$. The SBP and DBP of the experimental group after intervention were lower than those before intervention, and the difference was statistically significant $(\mathrm{P}<0.001)$. There was no significant difference between SBP and DBP in the control group before and after intervention $(\mathrm{P}>0.05)$.

Comparative analysis of the subgroup blood pressure drop before and after intervention of the experimental group: The experimental group was divided into a low-risk group, a middle-risk group, and a high-risk group according to cardiovascular risk stratification (Table 4). The decrease in blood pressure is shown in Table 5. SBP decreased by $8.77 \pm 5.57,10.80 \pm 8.16$, and $12.32 \pm 7.59 \mathrm{mmHg}$, respectively, $\mathrm{P}=0.402(\mathrm{P}>0.05)$. The difference was not statistically significant. DBP decreased by $4.69 \pm 5.22,6.40 \pm 5.26$, and $5.45 \pm 5.49 \mathrm{mmHg}$, respectively, $\mathrm{P}=0.628(\mathrm{P}>0.05)$. The difference was not statistically significant.
Comparison and analysis of compliance in the two groups: Compliance of hypertension treatment was evaluated according to the CPAT score reported by Morisky et al. CPAT scores are shown in Table 6. There was no significant difference in CPAT scores between the experimental group and the control group before intervention. After intervention, the CPAT score in the experimental group was higher than that in the control group, with a statistically significant difference $(\mathrm{P}<0.05)$. CPAT scores in the experimental group after intervention were higher than that before intervention $(\mathrm{P}<0.05)$. There was no significant difference in CPAT scores before and after intervention in the control group $(\mathrm{P}>0.05)$.

Comparison of HPSMBRS in the two groups: Hypertension self-management behaviors were measured by the HPSMBRS for hypertension patients designed by Zhao et al. (22). HPSMBRS scores of the two groups before and after intervention are shown in Table 7. There was no significant difference in HPSMBRS scores between the experimental group and the control group before 
Table 6 Comparison of CPAT score in the two groups

\begin{tabular}{|c|c|c|c|c|}
\hline Group & \multicolumn{4}{|c|}{ CPAT score } \\
\hline Experimental group & $2.28 \pm 0.56$ & $3.10 \pm 0.68$ & -7.20 & $<0.05$ \\
\hline Control group & $2.35 \pm 0.55$ & $2.38 \pm 0.64$ & -3.07 & 0.760 \\
\hline $\mathrm{t}$ & -0.663 & 5.937 & & \\
\hline
\end{tabular}

CPAT, Colorado Pretrial Assessment Tool.

Table 7 Comparison of HPSMBRS score between the two groups

\begin{tabular}{|c|c|c|c|c|}
\hline Group & \multicolumn{4}{|c|}{ HPSMBRS score } \\
\hline Experimental group & $72.27 \pm 5.94$ & $74.57 \pm 5.84$ & -6.657 & $<0.001$ \\
\hline Control group & $70.82 \pm 5.67$ & $70.85 \pm 5.78$ & -0.084 & 0.933 \\
\hline $\mathrm{t}$ & 1.367 & 3.502 & & \\
\hline
\end{tabular}

HPSMBRS, Hypertension Patients Self-Management Behavior Rating Scale.

intervention $(\mathrm{P}>0.05)$. After intervention, the HPSMBRS score in the experimental group was higher than that in the control group, with a statistically significant difference $(\mathrm{P}<0.05)$. HPSMBRS scores in the experimental group after intervention were higher than that before intervention $(\mathrm{P}<0.05)$. There was no significant difference in HPSMBRS scores before and after intervention in the control group $(\mathrm{P}>0.05)$.

Comparison of blood lipid, BMI and urinary microalbumin between the two groups before and after intervention: BMI, urinary microalbumin, TC, LDL-C, HDL-C and TG before and after intervention in the experimental group and the control group were shown in Table 8. There was no significant difference in BMI, urinary microalbumin, TC, LDL-C, HDL-C and TG between the experimental group and the control group before intervention $(\mathrm{P}>0.05)$. After intervention, there was no significant difference in BMI, urinary microalbumin, TC, LDL-C, HDL-C and TG between the experimental group and the control group $(\mathrm{P}>0.05)$. In the experimental group, BMI, urinary microalbumin, TC, LDL-C after intervention was lower than that before intervention, with statistically significant differences $(\mathrm{P}<0.05)$, while HDL-C and TG before and after intervention showed no significant differences $(\mathrm{P}>0.05)$. In the control group, there were no significant differences in BMI, urinary microalbumin, TC, LDL-C, HDL-C and TG before and after intervention $(\mathrm{P}>0.05)$.

\section{Discussion}

This experiment verifies the effectiveness of the new mode of patient management through mobile platforms. The study showed that the combination of medical intervention and mobile network platform in the experimental group could improve patient compliance and reduce blood pressure, while there was no significant difference in blood pressure drop values before and after intervention in the three subgroups. It is suggested that the combination of medical intervention and mobile platform can improve the management effect of hypertension patients, and is equally effective for low-risk, medium-risk, and high-risk patients, and can improve the control rate and compliance of hypertension.

The new mode of mobile-platform patient management is effective for the management of patients with hypertension, as it contributes to reducing the patients' blood pressure while improving the patients' compliance, awareness and level of self-management, and satisfaction. This is consistent with the research results of Shi et al. (23). 
Table 8 Comparison of blood lipid, BMI and urinary microalbumin between the two groups

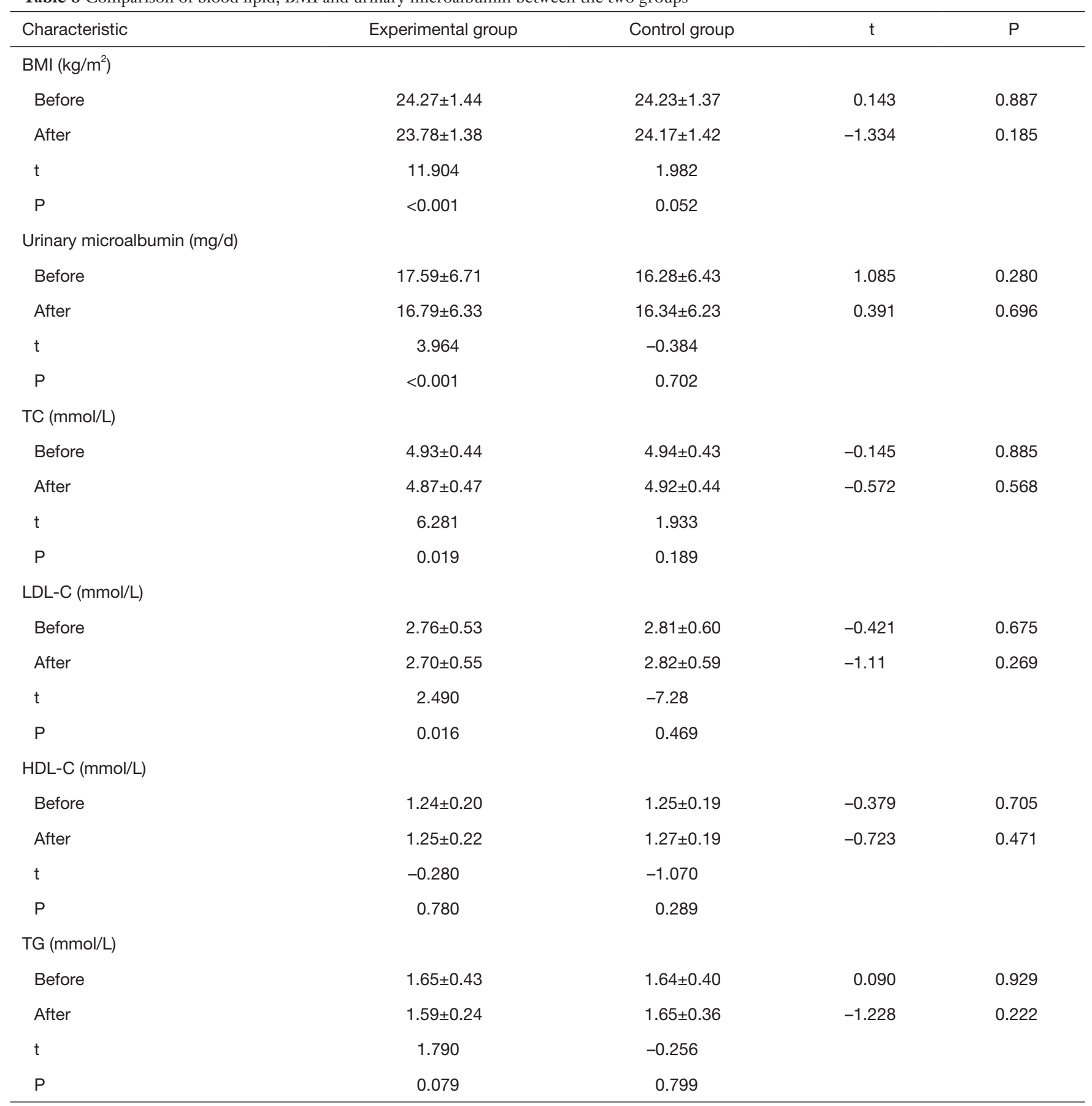

This shows that the new model's mobile platform is feasible and effective for hypertension management. The model not only reduces the patient's blood pressure value and reduces the damage to important organs but also improves the patient's understanding of the disease and the treatment compliance, promotes the improvement of lifestyle, better manages the blood pressure, and slows the development of the disease. In the subgroup analysis, the SBP and DBP of the low-risk group, the medium-risk group and the highrisk group after intervention were all lower than before 
intervention, but the difference between the groups was not statistically significant $(\mathrm{P}>0.05)$. This shows that the conventional hypertension management combined with the new mode of mobile network platform (WeChat) has the same effect on hypertension management of different risk levels and is equally effective.

The new management mode manages hypertension patients through a mobile medical platform, which can save medical resources and reduce the burden on local medical services (23). Patients spend most of their time managing themselves at home, reducing the burden on hospitals, and allowing more resources for patients who need them. At the same time, through the communication between family members, it also indirectly improves the awareness of the patients' family members of the disease, promotes the improvement of lifestyle, and thus reduces the medical burden for the whole family. Once the patient encounters a problem, they can contact the doctor directly, adjust the medical plan, and make an appointment with the doctor to reexamine. It can greatly improve the initiative of patients and their families in disease control and disease management. At the same time, it can also increase the communication between doctors and patients, ease the tension between doctors and patients caused by various social parties, reduce medical costs, improve service efficiency, and increase patient satisfaction (24).

The new management mode combining traditional medical treatment and mobile medical treatment management is still in its infancy in China. In addition, the unbalanced and unreasonable distribution of medical resources has limited the promotion of the new management mode. When compared with foreign countries, the shortage of medical personnel in China and the large clinical workload limits the promotion of mobile medical management. Patients with different characteristics have different levels of acceptance and use of mobile information. For the elderly, the knowledge of how to use mobile devices is a challenge. Age, education level, disease status, and home addresses all affect the promotion and application of mobile medical treatments. In Zhang's study, the effects of hypertension control were evaluated through self-designed questionnaires, blood pressure, and risk factor controls (25). Wang evaluated the results through a self-designed questionnaire and blood pressure results (26). There is a current lack of a complete evaluation and management system. In our country, network infrastructure construction is not widespread, which affects the implementation of mobile information technology. Inadequate infrastructure is a challenge, especially in rural areas. It is important for the state to prioritize the construction and maintenance of network infrastructure and ensure the stability of networks in different regions.

This study has limitations. First, mobile health interventions are potentially risky, and patients are less likely to return to treatment when they need to. WeChat intervention may give a false diagnosis or guidance and delay regular follow-ups. However, our researchers maintained close contact with patients to provide correct guidance and timely correction to minimize these risks. Second, due to the limitation of research funds and human resources, only three months of clinical efficacy observation has been conducted, and further follow-up studies will be conducted at a later period.

\section{Conclusions}

To sum up, the new management mode combining traditional medical treatment with mobile medical treatment can improve the effective management of hypertension patients. It is beneficial to improve patient medication compliance, enhance self-management ability, and improve healthy lifestyles.

\section{Acknowledgments}

We would like to thank all the medical workers in the Department of Cardiology, The First Hospital of Shanxi Medical University for their supports and cooperation. Thanks to the master students of the department for their assistance in collecting data.

Funding: None.

\section{Footnote}

Reporting Checklist: The authors have completed the CONSORT reporting checklist. Available at http://dx.doi. org/10.21037/cdt-20-589

Data Sharing Statement: Available at http://dx.doi. org/10.21037/cdt-20-589

Conflicts of Interest: All authors have completed the ICMJE uniform disclosure form (available at http://dx.doi. org/10.21037/cdt-20-589). The authors have no conflicts of 
interest to declare.

Ethical Statement: The authors are accountable for all aspects of the work in ensuring that questions related to the accuracy or integrity of any part of the work are appropriately investigated and resolved. The study was conducted in accordance with the Declaration of Helsinki (as revised in 2013). The study was approved by ethics board of The First Hospital of Shanxi Medical University (No. sxykdxdyyyll201964) and informed consent was taken from all the patients.

Open Access Statement: This is an Open Access article distributed in accordance with the Creative Commons Attribution-NonCommercial-NoDerivs 4.0 International License (CC BY-NC-ND 4.0), which permits the noncommercial replication and distribution of the article with the strict proviso that no changes or edits are made and the original work is properly cited (including links to both the formal publication through the relevant DOI and the license). See: https://creativecommons.org/licenses/by-nc-nd/4.0/.

\section{References}

1. Lim SS, Vos T, Flaxman AD, et al. A comparative risk assessment of burden of disease and injury attributable to 67 risk factors and risk factor clusters in 21 regions, 1990-2010: a systematic analysis for the Global Burden of Disease Study 2010. Lancet 2012;380:2224-60.

2. Mozaffarian D, Benjamin EJ, Go AS, et al. Heart disease and stroke statistics--2015 update: a report from the American Heart Association. Circulation 2015;131:e29-322.

3. Yusuf S, Reddy S, Ounpuu S, et al. Global burden of cardiovascular diseases: Part II: variations in cardiovascular disease by specific ethnic groups and geographic regions and prevention strategies. Circulation 2001;104:2855-64.

4. Kearney PM, Whelton M, Reynolds K, et al. Global burden of hypertension: analysis of worldwide data. Lancet 2005;365:217-23.

5. National Health Commission of the People's Republic of China. Report on the Status of Nutrition and Chronic Diseases of Chinese Residents (2015). Beijing: People's Medical Publishing House, 2015.

6. Lu J, Lu Y, Wang X, et al. Prevalence, awareness, treatment, and control of hypertension in China: data from 1.7 million adults in a population-based screening study (China PEACE Million Persons Project). Lancet 2017;390:2549-58.
7. Wang Z, Chen Z, Zhang L, et al. Status of Hypertension in China: Results From the China Hypertension Survey, 2012-2015. Circulation 2018;137:2344-56.

8. Bengtsson U, Kasperowski D, Ring L, et al. Developing an interactive mobile phone self-report system for self-management of hypertension. Part 1: patient and professional perspectives. Blood Press 2014;23:288-95.

9. Zhang LL. Effect of Community-based Self-management Program among Hypertension Patient. China: Chinese Center for Disease Control and Prevention, 2010.

10. Zhang PJ, Tan J. Application of internet technology in health management of chronic kidney disease: a literature review. Journal of Nursing Science 2017;32:99-102.

11. Burke LE, Ma J, Azar KM, et al. Current Science on Consumer Use of Mobile Health for Cardiovascular Disease Prevention: A Scientific Statement From the American Heart Association. Circulation 2015;132:1157-213.

12. Muralidharan S, Mohan V, Anjana RM, et al. Mobile Health Technology (mDiab) for the Prevention of Type 2 Diabetes: Protocol for a Randomized Controlled Trial. JMIR Res Protoc 2017;6:e242.

13. Hou J, Ndasauka Y, Pan X, et al. Weibo or WeChat? Assessing Preference for Social Networking Sites and Role of Personality Traits and Psychological Factors. Front Psychol 2018;9:545.

14. CNNIC (2015). China Internet Network Information Center the Thirty-Sixth Statistics Report of China Internet Development.

15. Zhu D, He Y, Tian F. Social life within HERE: how SNS empowers different generations in China. Beijing: Social Sciences Academic Press (China), 2018.

16. Zhang $\mathrm{X}$, Wen D, Liang J, et al. How the public uses social media wechat to obtain health information in china: a survey study. BMC Med Inform Decis Mak 2017;17:66.

17. Zeng F, Deng G, Wang Z, et al. WeChat: a new clinical teaching tool for problem-based learning. Int J Med Educ 2016;7:119-21.

18. Chinese Hypertension Guideline Revision Committee; Hypertension Alliance (China); Hypertension Committee of Chinese Physicians Association, Chinese Society of Cardiology, Chinese Medical Association; Hypertension Branch of Chinese Association for International Exchange of Medical Care; Hypertension Branch of Chinese Geriatric Society. 2018 Chinese guidelines for the management of hypertension. Chinese Journal of Cardiovascular Medicine 2019;24:24-56.

19. Chinese Hypertension Prevention and Treatment Guideline Revision Committee. 2018 revision of Chinese 
hypertension prevention and treatment guidelines. Prev

Treat Cardio-Cerebral-Vascular Dis 2019;19:1-44.

20. Bittar N. Maintaining long-term control of blood pressure: the role of improved compliance. Clin Cardiol 1995;18:III-16.

21. Morisky DE, Green LW, Levine DM. Concurrent and predictive validity of a self-reported measure of medication adherence. Med Care 1986;24:67-74.

22. Zhao QL, Liu X. Reliability and Validity of the Hypertension Patients Self-Management Behavior Rang Scale (HPSMBRS). Chinese Nursing Management 2012;12:26-31.

23. Shi J, Wang P, Wang AM. The Clinical Study of a New

Cite this article as: Sun YQ, Jia YP, Lv JY, Ma GJ. The clinical effects of a new management mode for hypertensive patients: a randomized controlled trial. Cardiovasc Diagn Ther 2020;10(6):1805-1815. doi: 10.21037/cdt-20-589
Management Model Using Mobile Health for Senile Hypertension. Health 2019;11:1087-94.

24. Zhang F. Discussion on the self-management mode of community elderly hypertension patients participated by higher vocational nursing students. Health Vocational Educ 2017;35:78-9.

25. Zhang YF, Jiang XJ, Zhou HJ, et al. Significance of remote information exchanging platform to improve the goal attainment rate of hypertension patients. Chin J Health Manage 2016;10:308-12.

26. Wang PP, Ying YP, Gao ZL, et al. Application of APP in continuous nursing of patients with type 2 diabetes and hypertension. Hainan Medical Journal 2016;27:2057-60. 